\title{
FAST NEUTRON RADIOTHERAPY FOR LOCALLY ADVANCED PROSTATE CANCER:
}

RESULTS OE AN RTOG RANDOMIZED STUDY ${ }^{+}$

George E. Laramore, Ph.D., M.D a , John M. Krall, Ph.g. b, Frank J. Thomas, M.D. ${ }^{\prime}$, Thomas W. Griffin, M.D. , Moshe H. Maor, M.D. and Frank R. Hendrickson,
M.D.

a Department of Radiation Oncology, University of Washington Hospital, Seattle, WA 98195

b

Statistical Division, Radiation Therapy Oncology Group, 925 Chestnut Street, Philadelphia, PA 19167

$C$ Department of Radiation Therapy, Cleveland Clinic Foundation, Cleveland, $\mathrm{OH}$ 44106

d Department of Radiation Therapy, 1.D. Anderson Hospital, Houston, TX 77030

e Neutron Therapy Department, Fermi National Accelerator Laboratory, Batavia, IL 68511 and Department of Therapeutic Radiology, Rush-Presbyterian-St. Luke's Medical Center, Chicago, IL 60612

Running head: Neutron Radiotherapy for Prostate Cancer

Address for reprints: G.E. Laramore, Ph.D., M.D. Department of Radiation Oncology University of Washington Hospital RC-ø8 Seattle, WA 98195

+ Supported by the National Cancer Institute under core grant $\mathrm{CA}-23113$ and individual facility grants $\mathrm{CA}-12441, \mathrm{CA}-17465, \mathrm{CA}-18081$ and $\mathrm{CA}-19720$. 
ABSTRACT

Between June, 1977 and April, 1983, the Radiation Therapy Oncology Group (RTOG) sponsored a Phase III randomized study investigating fast neutron radiation therapy in treatment of patients with locally advanced (stage $C$ and $D_{1}$ ) adenocarcinoma of the prostate gland. Patients were randomized to receive either conventional photon radiation therapy or fast neutron irradiation used in a mixed-beam treatment schedule (neutron/photon). The randomization was purposefully unbalanced in favor of the experimental treatment $(60 \%-40 \%)$. A total of 91 eligible patients were entered in the study. The two treatment groups were balanced in regards to all major prognostic variables.

The overall local/regional tumor recurrence rate for the mixed-beam-treated group of patients is $7 \%$. The overall local/regional tumor recurrence rate for the photon (x-ray) treated group of patients is $22 \%$. The difference is statistically significant at the $p=0.65$ level. The 5-year survival rate for the mixed-bean-treated group is $62 \%$. The 5-year survival rate for the photontreated group is 358 . This difference is also statistically significant $(p<0.05)$

This study demonstrates that a regional treatment modality, in this case mixedbeam irradiation, can influence both local/regional tumor control and survival in patients with locally-advanced adenocarcinoma of the prostate gland. 
INTRODUCTION

Prostate cancer is the second most common malignancy in males in the United States (excluding non-melanoma skin cancer). In 1984, the American Cancer Society estimates there will be 76,000 new cases of prostate cancer and 25,000 deaths due to the disease. ${ }^{1}$ It characteristically occurs in elderly patients, and in many cases it is only slowly progressive and requires no treatment. Early stage ${ }^{2} A_{1}$ lesions often are given no treatment at all. More advanced $A_{2}$, $\mathrm{B}_{1}$ and $\mathrm{B}_{2}$ lesions are usually treated either with radical surgery or photon radiation therapy with representative series of each treatment method showing about the same 5 and 10 year survival rates. Patients presenting with distant metastases are treated in a palliative fashion with radiation therapy and/or hormonal therapy.

An area of current controversy is the treatment of patients presenting with stage $C$ and $D_{1}$ disease--either tumor extending through the capsule of the gland, invading into adjacent structures or accompanied by positive pelvic lymph nodes. Standard photon radiation therapy has been recommended as the treatment of choice, but until now it has not been definitively proven that any local/regional treatment is capable of altering the survival of patients with these stages of disease. In this work, we will show that improved local/regional control of stage $C$ and $D_{1}$ disease as achieved with mixed-beam irradiation does lead to improved patient survival. This report describes the results of a Radiation Therapy Oncology Group (RTOG) study which compares megavoltage photon radiation therapy against fast neutrons used in a mixed-beam (neutron/photon) treatment schedule for locally advanced (Stages $C$ and $D_{1}$ ) 
prostate cancer. Both local/regional tumor control and survival were improved (statistically significant at $\underline{p} \underline{\emptyset .05)}$ with the mixed-beam method of treatment.

Fast neutron radiation therapy has been predicted to be advantageous in the treatment of prostate cancer for a number of reasons related to the high linear energy transfer (LET) of the fast neutron in tissue. Fast neutrons typically deposit $20-10$. times more energy in matter than megavoltage $\mathrm{X}$-rays, and this high LET gives rise to the radiobiological properties of potential importance in clinical cancer treatment. ${ }^{3}$ High LET radiations are less dependent on the presence of oxygen to accomplish their cell-killing effect than are low LET Xrays and, thus, are more effective killers of the hypoxic cells found in large tumor masses. The oxygen enhancement ratio (OER) for fast neutrons is approximately 1.6 compared to an OER of $2.5-3.0$ for high energy photons. Furthermore, the type of radiation damage inflicted by neutrons is less readily repaired by tumor cells. There is a reduced ability to repair sublethal damage manifested by a decreased shoulder on cell survival curves, and there is a reduced ability to repair potentially lethal damage which could be especially important for slowly growing tumors such as prostate cancer with a large fraction of cells in $a G_{O}$ or "resting" phase. There is also less variation in radiosensitivity across the cell cycle with neutrons than with conventional $\mathrm{X}$ rays. These radiobiological properties, along with the work of Batterman et al. ${ }^{4}$ indicating a higher relative biological effectiveness (RBE) for neutrons over photons in slow growing, better differentiated photonradioresistant tumors, all suggest that neutrons should show an improvenent over photons in the treatment of prostate cancer. 


\section{MATERIALS AND METHODS}

Ninety-one eligible patients were accrued to the study between June, 1977 and April, 1983. All patients had either stage $C$ or $D_{1}$, biopsy-proven adenocarcinoma of the prostate gland. Mandatory pre-randomization evaluation included a complete history and physical examination, chest X-ray, complete blood count, blood chemistries including liver function tests, serum calcium, alkaline phosphatase, acid phosphatase and a radionucleide bone scan. Bipedal lymphangiography and/or exploratory laparotomies were performed on a total of 41 patients. CT scans of the pelvis were performed in about half the cases. To be eligible for randomization, patients had to be less than 80 years of age, have an initial Karnofsky performance score greater than $4 \emptyset$, could not have had prior pelvic irradiation or extensive prior pelvic surgery, and could not have had a prior history of cancer (excluding non-melanoma skin cancers). Prior hormonal therapy was allowed but had to be adequately documented. Informed consent was given by all patients who entered the study.

Patients were randomized through the RTOG operational office to receive one of two possible methods of treatment: 1) photon (control) radiation therapy, or 2) mixed-beam irradiation. Mixed-beam irradiation is a mixture of $2 / 5$ neutrons and $3 / 5$ photons. The randomization was purposefully unbalanced (60\%$49 \%)$ in favor of the experimental treatment.

Photon-treated patients were to receive a dose of $5 \emptyset \mathrm{Gy}$ to the whole pelvis (prostate and nodes) at a dose rate of 1.8 to 2 Gy per fraction followed by a 20 Gy boost dose to the prostate and any areas of proven bulky extra-prostatic 
disease. Mixed-beam-treated patients were to receive a dose of $5 \emptyset$ Gy photonequivalent (neutron dose multiplied by the institutional RBE plus the photon dose) mixed-beam irradiation to the whole pelvis, followed by a 20 Gy photonequivalent boost dose of mixed-beam irradiation to the prostate and any areas of proven bulky extra-prostatic disease, at a dose rate of 1.8 to 2 Gy photonequivalent per fraction. All patients were to be treated 5 days per week. Mixed-beam patients were to receive neutrons two days per week and photons three days per week. The radiation dose to the entire bladder was restricted to 60 Gy photons or photon-equivalent, the dose to the posterior rectal wall was restricted to $55 \mathrm{~Gy}$ photons or photon-equivalent, and the dose to the small bowel was limited to $55 \mathrm{~Gy}$ photons or photon-equivalent.

The following neutron treatment facilities participated in the study: the University of Washington, the Great Lakes Neutron Treatment Association, the M.D. Anderson facility at Texas A \& M University, and the Eermilab. Neutron doses were scaled according to the RBE's for the various institutions: 3.3 for the University of Washington and the Great Lakes Neutron Treatment Association, 3.I for M.D. Anderson, and 3.0 for the Eermilab. The garma contaminant was included in the neutron dose. The experimental treatment was designed to use a neutron/photon combination rather than neutrons alone because of the poorly penetrating physics characteristics of the neutron beams available at most of the facilities.

Portal films were required for each treatment field as were computer isodose calculations through the central axis plane of the pelvis and also through the prostate. All treatment parameters and patient records were reviewed by the study chairman. 
Patients were evaluated in follow-up at monthly intervals for the first three months after treatment, at three-month intervals for the remainder of the next three years, and every six months thereafter.

Based on the chi-square test of independence, the two groups were balanced according to age distribution, tumor grade (Mostofi schene ${ }^{5}$ ), stage (C vs. $D_{1}$ ), method of diagnosis (TURP vs. needle biopsy), percent of patients having lymphangiograms, laparotomies or other methods of nodal evaluation, initially elevated acid phosphatase levels, degree of seminal vesicle involvement, Karnofsky performance status, race, prior hormonal therapy, cardiac disease status, other intercurrent disease status, and Gleason score. ${ }^{6}$ The presence of concomitant, benign prostatic hypertrophy was unbalanced at a marginally significant level $(p=\emptyset .06)$ and occurred most frequently in the inixed-beam treatment group.

RESULTS

The major endpoints of this study are local/regional tumor control and survival. Complication rates and tolerance of the irradiated normal tissues are secondary endpoints. The plots in this section are calculated using the actuarial method ${ }^{7}$, with times measured from the initiation of treatment.

Figure 1 shows the fraction of patients exhibiting a local/regional tumor control as a function of time. In the analysis of local control, a posttreatment abnormality was assumed to be of unknown significance in the period immediately following irradiation and was not counted as a failure until progression was noted. This method of analysis was chosen because prostate 
cancer is slow to respond to radiation therapy and often does not regress completely until several months after treatment is finished. For this reason the curves in Figure 1 start at the $100 \%$ level.

The overall local/regional recurrence rates are $7 \%$ for the mixed-beam-treated group of patients compared to $22 \%$ for the photon-treated group of patients. The difference, is statistically significant at the $p=0.05$ level.

Figure 2 shows the percent of patients surviving as a function of time. The 5year survival rate is significantly better for the mixed-beam-treated group of patients compared to the photon-treated group of patients $(p<0.05)$. Sixty-two percent of the mixed-bean-treated patients survived 5 years compared to $35 \%$ of the photon-treated. patients.

Measurable distant metastases were documented in $36 \%$ of the mixed-beam-treated patients and $44 \%$ of the photon-treated patients. This apparent difference fails to achieve statistical significance.

Treatment associated complications in most cases were mild and were predominately the expected side effects of nausea, diarrhea, dysuria and urinary urgency. Because of the poorly penetrating physics qualities of the neutron beams, skin and subcutaneous reactions were more common in the mixedbeam-treated group of patients. The incidence of severe complications was not significantly different between the two treatment groups and was $9 \%$ in the mixed-beam-treated group and $7 \%$ in the photon-treated group. The only fatal complication was a severe proctitis requiring surgical intervention that occurred in a photon-treated patient. 


\section{DISCUSSION}

Carcinoma of the prostate gland is a significant public health problem in the United states. As pointed out in the "Introduction" section, it is the second most cormon malignancy in males in this country. It kills approximately 25,000 people each year. In a prospective, randonized study comparing mixed-beam radiation therapy against what was judged to be the best conventional treatment (high dose photon radiation therapy) for advanced (stage $C$ and $D_{1}$ ) disease, the mixed-beam-treated group of patients did significantly better $(p \leq \emptyset .05)$ than the photon-treated group in terms of both local/regional tumor control and survival. The two treatment groups were matched as to all of the variables currently thought to be of relevance for this tumor systen.

Most. of the patients entered on this study were staged as stage $C$, but $5 / 36$ patients on the photon randomization and 6/55 patients on the mixed-beam randomization had proven metastases to the pelvic lymph nodes. No doubt the frequency of occult nodal disease was substantially higher. Operative staging series of clinical stage $C$ patients show an incidence of nodal involvenent ranging from $40-60 \% .8,9,10$ Eurthermore, in one series ${ }^{8} 29 \%$ of the nodal metastases occurred only in the obturator and hypogastric nodal groups--regions that are not well visualized with bipedal lymphangiography. It may be that the observed survival difference was due in part to the ability of the mixed-beam treatment to more frequently sterilize nodal disease in the pelvis.

The clinically assessed local/regional failure rate on this study was $22 \%$ with the photon treatment and $7 \%$ with the mixed-beam treatment. Local/regional failure was defined as one or more of the following: the product of the tumor 
diameters $>258$ larger than the "on-study" values, a positive biopsy at a time greater than two years from completion of treatment, a new soft tissue extension of tumor, or pelvic nodes becoming positive. The majority of patients entered on this study had extremely advanced local disease.

Neglia et al. ${ }^{11}$ report a local/regional failure rate of $5.2 \%$ for early stage C lesions and $15.1 \%$ for advanced stage $C$ lesions. Perez et al. ${ }^{12}$ report local failure rates of $20 \%$ and $12 \%$ respectively for patients with stage $C$ lesions treated with either $65-70$ Gy or $>70$ Gy total radiation dose to the prostate. Rangala et al. ${ }^{13}$ report a local failure rate of $24 \%$ for a mixed group of stage $\mathrm{B}$ and $\mathrm{C}$ patients treated with definitive radiotherapy. The Patterns of Care study $^{14}$ demonstrated a $19 \%$ local failure rate in patients with stage $C$ lesions who recieved $>65$ Gy. Hence, the local/regional control exhibited by our photon-treated patients is comparable with that previously reported for patients with stage $C$ disease.

Survival is a more concrete parameter than is 1ocal/regional tumor control. The 5-year survival rates were $62 \%$ for the mixed-beam-treated patients vs. $35 \%$ for the photon-treated patients. Patient survival is dependent on many variables and has been reported in the literature $12,15,16$ to be as high as 55$60 \%$ at 5 years. Neglia et al. ${ }^{11}$ show a definite dependence of survival on primary tumor size, with less advanced stage $C$ lesions having a 5-year survival of approximately $60 \%$ compared with approximately $45 \%$ for more advanced stage C lesions. The majority of our patients had extremely advanced local disease.

A stepwise cox analysis ${ }^{17}$ has been used to identify the important patient parameters relating to survival in this study (Table 1). Age, stage of lesion 
and whether or not serum acid phosphatase levels were initially elevated were important parameters associated with survival; however, these parameters proved to be less important predictors of survival than form of treatment. The most important predictor of survival was whether or not patients were treated with mixed-beam irradiation $(p<\emptyset . \emptyset 1)$.

The results of this study demonstrate that a local/regional form of treatment can indeed affect survival rates in patients with locally advanced prostate cancer. A new generation of neutron treatment facilities is nearing completion and will greatly improve the technical ability of investigators to treat deeply-seated tumors. The advantageous physics characteristics of the treatment beams generated at these facilities will allow treatment with neutrons alone instead of the mixed-beam irradiation used in this study, and offer the potential to further improve the results of treatment of locally advanced prostate cancer. In any case, if the present study continues to demonstrate improved local/regional tumor control and survival at longer follow-up times, it will be important to extend this work to less advanced stage $\mathrm{A}_{2}$ and $\mathrm{B}$ lesions. 
Table I. Important prognostic variables* relating to patient survival as determined by a multi-variant Cox step-wise analysis ${ }^{17}$ for 79 patients for whom all factors are known.

Independent Variables

Treatment

(Mixed-beam vs. photons)

Age

Stage (C vs. $D_{1}$ )

Elevated Serum Acid Phosphatase

\begin{tabular}{cr} 
P-Value for Improvement & Rank \\
\hline$p<0.01$ & lst \\
$p<0.05$ & 2nd \\
$p<0.05$ & 3rd \\
$p=0.06$ & 4th
\end{tabular}


REFERENCES

1. Silverberg E. Cancer statistics, 1984. Ca-A Cancer Journal for Clinicians $1984 ; 34: 7-23$.

2. The American Urological Staging System will be used in this manuscript. A concise review of this system and its relation to the AJC TMN staging system may be found in Rubin P. Clinical Oncology-A Multidisciplinary Approach, 6th Ed., American Cancer Society 1983:210-11.

3. For a general review see Hall EJ. Radiobiology for the Radiologist, 2nd edition, Hagerstown, Harper \& Row, 1978.

4. Batterman JJ, Breur K, Hart GAM, and van Peperzeel HA. Observations on pulmonary metastases in patients after single doses and multiple fractions of fast neutrons and cobalt-60 gamma rays. Europ J Cancer 1981; 17:53948.

5. Mostofi FK. Grading of prostate cancer. Cancer Chemother Rep 1975; $59: 111-17$.

6. Gleason DF, Mellinger FT. Veterans Administration Cooperative Urological Research Group: Prediction of prognosis for prostatic adenocarcinoma by combined histological grading and clinical staging. J Urol 1974; 111:5864.

7. Kaplan EL, Meier P. Nonparametric estimation fron incomplete observations. J Am Stat Assoc 1958; 53:457-81.

8. McLaughl in AP, Saltzstein SL, MoCullough DL, Gittes RF. Prostatic carcinoma: incidence and localization of unsuspected lymphatic metastases. J Urol 1976; 115:89-94.

9. Baszell W, Beam MA, Hilaris BS, Whitmore WE. Prostatic adenocarcinoma: relationship of grade and local extent to the pattern of metastases. J Urol 1977; 118:278-82. 
10. Flocks RN, O'Donoghue EPN, Milleman LA, Culp DA. Surgery of prostatic cancer. Cancer 1975; 36:705-17.

11. Neglia WJ, Hussey DH, Douglas DE. Megavoltage radiation therapy for carcinoma of the prostate. Int $J$ Rad oncol Biol phys 1977; 2:873-82.

12. Perez CA, Walz BJ, Zivnuska FR, Pilepich M, Prasad K, Bauer W. Irradiation of carcinoma of the prostate localized to the pelvis: analysis of tumor response and prognosis. Int J Rad Oncol Biol Phys 1980; $6: 555-63$

13. Rangala $\mathrm{N}, \mathrm{Cox} \mathrm{J}$, Byhardt $\mathrm{RW}$, Wilson JF, Greenburg M, da Concicao AL. Local control and survival after external irradiation for adenocarcinoma of the prostate. Int J Rad Oncol Biol Phys 1982; 8:1909-14.

14. Leibel SA, Hanks GE, Kramer S. Patterns of care outcome studies: results of the national practice in adenocarcinoma of the prostate. Int $\mathrm{J}$ Rad Oncol Biol phys 1984; 10:401-9.

15. Harisiadas L, Veenema RJ, Senyszyn JJ, Puchner PJ, Tretter P, Romas NA, Chang CH, Lattimer JK, Tannenbaum M. Carcinoma of the prostate: treatment with external radiation therapy. Cancer 1978; 41:2131-42.

16. Taylor WJ, Richardson G, Hafferman MD. Radiation therapy for localized prostate cancer. Cancer 1979; 43:1123-27.

17. Cox DR. Analysis of Binary Data. London, Methuen and Co., 1970. 
FIGURE CAPTIONS

Figure 1: Time to clinically-assessed local/regional tumor progression. The dashed curve refers to the mixed-beam-treated subgroup, and the solid curve represents the photon-treated subgroup. The curves are calculated on an actuarial basis, and the difference is statistically significant at the $p=0.05$ level.

Figure 2: Patient survival as a function of time. The dashed curve represents the mixed-beam-treated subgroup, and the solid curve represents the photon-treated subgroup. Survival for the mixed-beam group is statistically significantly better at the $p<0.05$ level. 
Time to Local Recurrence as a Function of Treatment Option

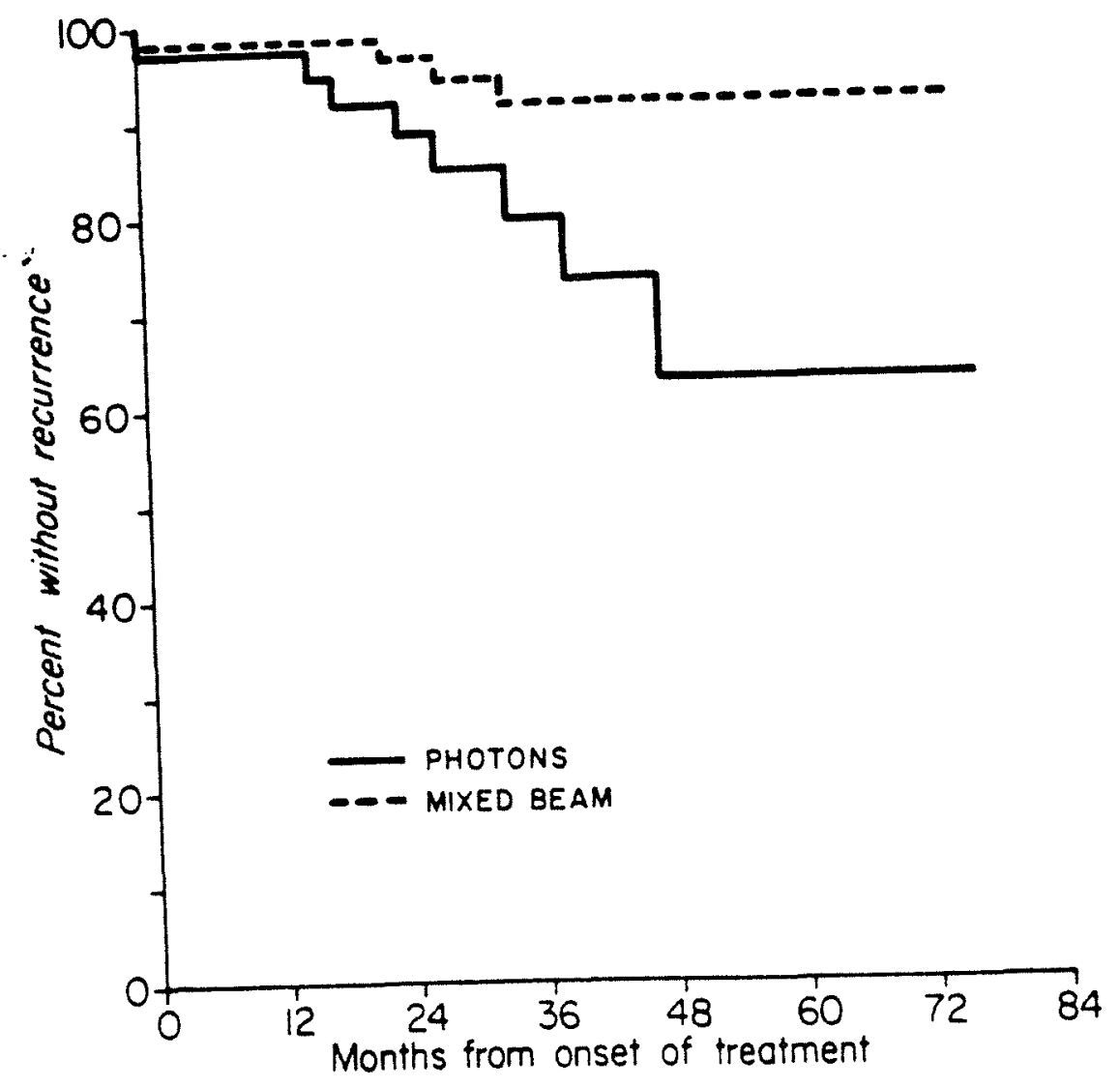




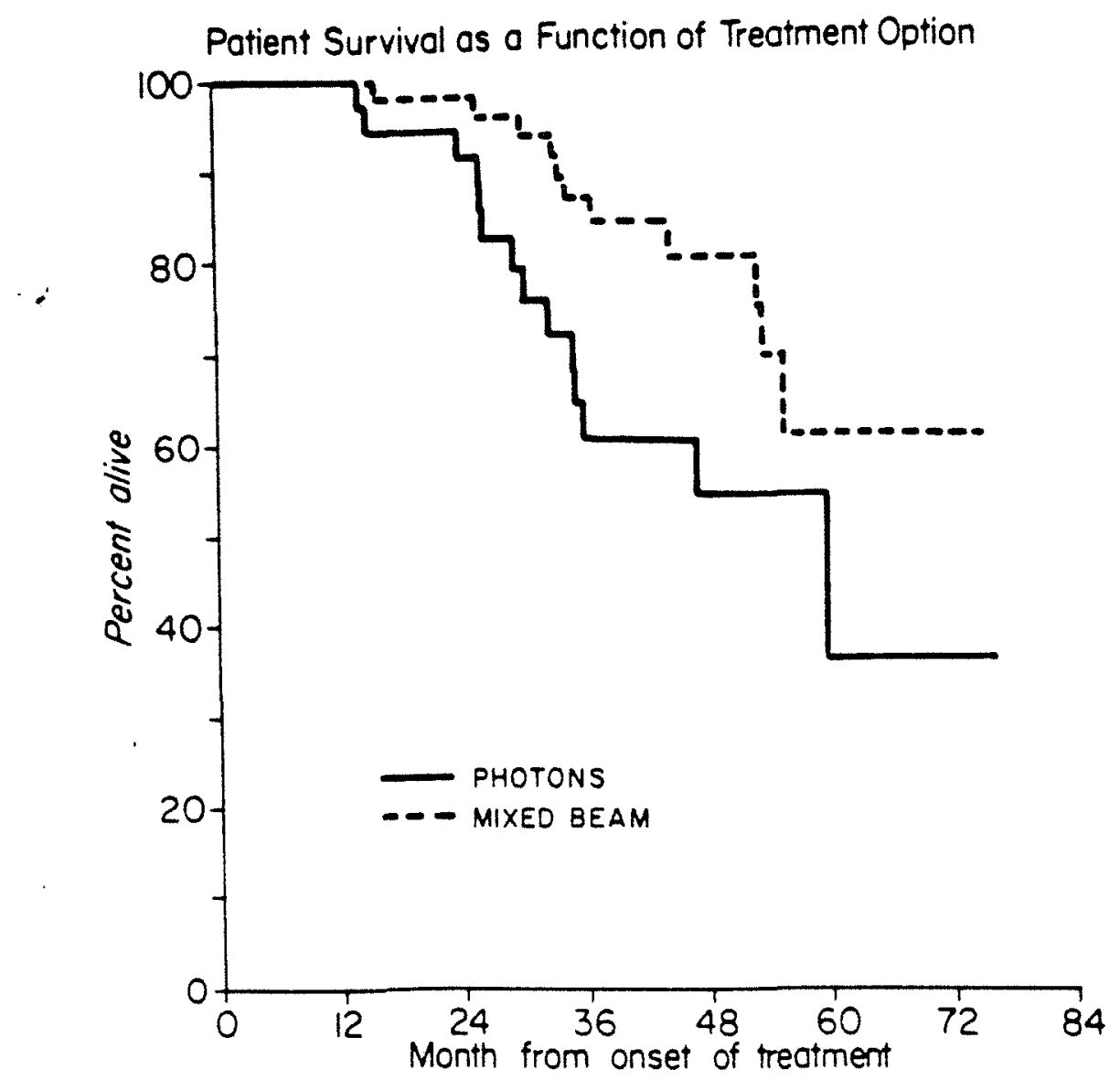

